\title{
Corrigendum: Probiotics Reduce Health Care Cost and Societal Impact of Flu-Like Respiratory Tract Infections in the USA: An Economic Modeling Study
}

\author{
Irene Lenoir-Wijnkoop ${ }^{1 *}$, Dan Merenstein ${ }^{2}$, Daria Korchagina ${ }^{3}$, Christa Broholm ${ }^{4}$, \\ Mary Ellen Sanders ${ }^{5}$ and Dan Tancredi ${ }^{6}$

\begin{abstract}
Department of Pharmaceutical Sciences, University of Utrecht, Utrecht, Netherlands, ${ }^{2}$ Family Medicine Department, Human Health Innovation, Hoersholm, Denmark, ${ }^{5}$ Dairy \& Food Culture Technologies, Centennial, CO, United States, CA, United States
\end{abstract} \\ Georgetown University, Washington, DC, United States, ${ }^{3}$ Real World Insights, IQVIA, Paris, France, ${ }^{4}$ Chr. Hansen AVS, \\ ${ }^{6}$ Department of Pediatrics and the Center for Healthcare Policy and Research, University of California, Davis, Sacramento,
}

Keywords: probiotics, health economics, respiratory tract infection, influenza, cost savings

\section{A Corrigendum on}

\section{OPEN ACCESS}

Edited and reviewed by: lleana Mardare,

Carol Davila University of Medicine and Pharmacy, Romania

*Correspondence:

Irene Lenoir-Wijnkoop P.I.Lenoir-Wijnkoop@uu.nI

Specialty section:

This article was submitted to Pharmaceutical Medicine and Outcomes Research,

a section of the journal

Frontiers in Pharmacology

Received: 10 September 2019 Accepted: 13 September 2019

Published: 11 October 2019

Citation: Lenoir-Wijnkoop I, Merenstein D, Korchagina D, Broholm C Sanders ME and Tancredi D (2019)

Corrigendum: Probiotics Reduce

Health Care Cost and Societal Impact of Flu-Like Respiratory Tract Infections in the USA: An Economic Modeling Study.

Front. Pharmacol. 10:1182. doi: 10.3389/fphar.2019.01182
Probiotics Reduce Health Care Cost and Societal Impact of Flu-Like Respiratory Tract Infections in the USA: An Economic Modeling Study.

by Lenoir-Wijnkoop I, Merenstein D, Korchagina D, Broholm C, Sanders ME and Tancredi D (2019) Front. Pharmacol. 10:980. doi: 10.3389/fphar.2019.00980

In the original article, there was an error. There is a mistake in the Funding statement. The mention that the funder, Chr. Hansen, was not involved in the writing of the manuscript is incorrect. A correction has been made to the Funding statement:

"This project was supported by an unrestricted grant from Chr. Hansen. The funder was not involved in the study design, collection, analysis, interpretation of data or the decision to submit it for publication"

Furthermore, there is a mistake in the Conflict of Interest Statement. The declaration that the author DM consulted Bayer and Pharmative was erroneously omitted. A correction has been made to the Conflict of Interest Statement:

"Author CB is an employee at Chr. Hansen. Author DK is employed by IQVIA. Author MS serves as an executive science officer for the International Scientific Association for Probiotics and Prebiotics. She also reports personal fees outside the submitted work from the following entities: International Scientific Association for Probiotics and Prebiotics, Pharmavite, CD Investments, Dannon, Danone USA, Yakult, California Dairy Research Foundation, Winclove BioSciences BV, Nestle, Williams Mullen, New Chapter, Dutch Mill, Clorox, Pfizer, Visalia Dairy Company, Procter \& Gamble, Kelley Drye \& Warren LLP, Kellogg, Trouw Nutrition, Kerry, JHeimbach LLC, General Mills, Probi, and Medscape. Author DM declares consulting for Bayer and Pharmavite. The remaining authors declare that the research was conducted in the absence of any commercial or financial relationships that could be construed as a potential conflict of interest." 
Lastly, there was a mistake in the Methods, section Model Inputs and Data Sources, sub-section Probiotic Effect. The RTI incidence and antibiotic prescription rate was provided in the incorrect unit. A correction has been made to the Methods, section Model Inputs and Data Sources, sub-section Probiotic Effect, paragraph one:

"The clinical effects of probiotics were obtained from the meta-analyses published by the YHEC (King et al., 2014) and Cochrane (Hao et al., 2015). These were used to conduct two independent scenario analyses comparing generalized probiotic use versus non-use, each based on different assumptions: YHEC showed a significantly shorter duration of -0.77 days $[-1.50$ to -0.04] on an average duration of 7.4 days per episode of RTI, among otherwise healthy children and adults taking probiotics compared to those taking placebo. The Cochrane study reported that probiotics significantly reduced RTI duration by 1.89 days [1.75 to 2.03] per episode of an average duration of 8.82 days and RTI incidence by $30 \%(\mathrm{RR}=0.70$ [0.50 to 0.84$])$
(Table 1). The authors also found a significant reduction of the antibiotic prescription rate of $35 \%$ ( $R R=0.65$ [0.45 to 0.94$]$ ), which was applied to both scenarios." Additionally, the YHEC meta-analysis studied the impact of probiotics on work absenteeism. The reported standardized mean difference (SMD) in the number of days absent from work was used to estimate the impact of probiotics on productivity loss. The Cochrane metaanalysis focused on unvaccinated individuals; therefore, no impact of probiotics sourced from the Cochrane meta-analysis was applied in vaccinated patients.

The authors apologize for these errors and state that they do not change the scientific conclusions of the article in any way.

Copyright (c) 2019 Lenoir-Wijnkoop, Merenstein, Korchagina, Broholm, Sanders and Tancredi. This is an open-access article distributed under the terms of the Creative Commons Attribution License (CC BY). The use, distribution or reproduction in other forums is permitted, provided the original author(s) and the copyright owner are credited and that the original publication in this journal is cited, in accordance with accepted academic practice. No use, distribution or reproduction is permitted which does not comply with these terms. 